\title{
47. A Condition for an Abelian Group to be a Free Abelian Group with a Finite Basis.
}

\author{
By Hidehiko YamaBE.
}

(Comm. by KunUGI, M.J.A., May 16, 1951.)

1. Lat $G$ be a countable abelian (additive) group. An integer-valued function $f(\xi, \eta)$ on $G \times G$ is said to be bilinear if

$$
f\left(\xi_{1}+\xi_{i}, \eta_{1}+\eta_{z}\right)=\sum_{i, j} f\left(\xi_{i}, \eta_{j}\right)
$$

for any elements $\xi_{i}, \eta_{j},(i, j=1,2)$.

Put

$$
G_{p}=\{p \xi ; \xi \in G\},
$$

where $p$ is a fixed integer.

Prof. Igusa conjectured that an abelian group $G$ is a free abelian group with a finite basis if a bilinear integer-valued function $f(\xi, \eta)$ is defined on $G \times G$ which vanishes only at the identity element of $G \times G$, and if $G / G_{p}$ is a finite group.

The purpose of this note is to give an affirmative answer. We shall prove the

Theorem. An abelian group $G$ satisfying the above conditions is a free abelian group with a finite basis.

2. From the above condition concerning the bilinear function $f(\xi, \eta)$ we can easily deduce that

(i) there does not exist an element of finite order,

(ii) there does exist only a finite number of elements which are the divisors of a fixed element.

An element is said to be prime if the element has not any divisor except itself. The set of prime elements $\left\{\xi_{i}\right\}$ is called a canonical system if for relative prime integers $a_{i}$ the element

$$
a_{1} \xi_{1}+a_{2} \xi_{s}+\cdots+a_{s} \xi_{s}
$$

is also prime. A subgroup which is spanned by a canonical system is clearly a free abelian group. We shall now prove the following lemma.

Lemma. Let $\Xi$ be a subgroup of rank $s-1$, spanned by a canonical system $\left\{\boldsymbol{\xi}_{1}, \boldsymbol{\xi}_{2}, \ldots, \boldsymbol{\xi}_{s-1}\right\}$. If $G \neq \Xi$ there exists a canonical system $\left\{\phi_{1}, \phi_{i}, \ldots, \phi_{s}\right\}$ such that the subgroup $\Phi$ spanned by the $\left\{\varphi_{i}\right\}$ contains $\Xi$ and the rank of $\Phi$ is equal to $s$. 
Proof of the Lemma. Lat $\xi_{s}$ be a prime element which is not contained in $\Xi$. If $\left\{\xi_{1}, \ldots, \xi_{s-1}, \xi_{s}\right\}$ is not a canonical system, then there exists a prime element $\eta_{1}$ such that for an integer $l_{1} \neq 1$ and for relative prime integers $a_{i}$

$$
a_{1} \xi_{1}+\cdots+a_{s} \xi_{s}=l_{1} \eta_{1} .
$$

We can construct a unimodular matrix $\left(a_{i j}\right)$ of order $s$ whose first row coincides with $\left(a_{1}, \ldots, a_{s}\right)$ and whose components are all integers.

Put

$$
a_{i 1} \xi_{1}+\cdots+a_{i s} \xi_{s}=l_{i} \eta_{i},
$$

where $\eta_{i}$ 's are all prime.

Then by simple calculations

$$
\begin{aligned}
\left(\operatorname{det}\left(a_{i j}\right)\right)^{2} \operatorname{det}\left(f\left(\xi_{i}, \xi_{j}\right)\right) & =\operatorname{det}\left(f\left(l_{i} \eta_{i}, l_{j} \eta_{j}\right)\right. \\
& =\left(\operatorname{IIl} l_{k}\right)^{2} \operatorname{det}\left(f\left(\eta_{i}, \eta_{j}\right)\right) .
\end{aligned}
$$

Or

We have

$$
\operatorname{det}\left(f\left(\xi_{i}, \xi_{j}\right)\right)=\left(I I l_{k}\right)^{2} \operatorname{det}\left(f\left(\eta_{i}, \eta_{j}\right)\right) .
$$

$$
\operatorname{det}\left(f\left(\xi_{i}, \xi_{j}\right)\right) \neq 0,
$$

because $\xi_{i}$ 's are linearly independent.

It follows that

$$
\left|\operatorname{det}\left(f\left(\xi_{i}, \xi_{j}\right)\right)\right|>\left|\operatorname{det}\left(f\left(\eta_{i}, \eta_{j}\right)\right)\right| \geqq 1 .
$$

If $\left\{\eta_{i}\right\}$ is not canonical we can construct a system $\left\{\zeta_{i}\right\}$ such: that

$$
\left|\operatorname{det}\left(f\left(\eta_{i}, \eta_{j}\right)\right)\right|>\left|\operatorname{det}\left(f\left(\zeta_{i}, \zeta_{j}\right)\right)\right| \geq 1 .
$$

Repeating this process we shall finally obtain a canonical system. $\left\{\phi_{1}, \ldots, \phi_{s}\right\}$ such that the subgroup $\Phi$ spanned by $\left\{\varphi_{i}\right\}$ contains: $\Xi$ and has a rank $s$.

3. Proof of the theorem.")

Put

$$
\Xi_{p}=\{p \xi ; \xi \in \Xi\}
$$

Then clearly

$$
\Xi_{p}=G_{p} \cap \Xi .
$$

By $x$ we denote the rank of $\Xi$. Then

1) The technic of this step is due to Prof. Igusa. The author's proof is more complicated. 


$$
\begin{aligned}
x^{p}=\text { the order of } \Xi / \Xi_{p} & =\text { the order of }\left(\Xi+G_{p}\right) / G_{p} \\
& \leqq \text { the order of } G / G_{p} .
\end{aligned}
$$

We see therefore the rank of such a $\Xi$ is bounded, and by the above lemma $G$ must coincide with one of such a $\Xi$.

Remark 1. It is true that without the condition that $G / G_{p}$ is a finite group, $G$ should be a free abelian group of infinite rank. The proof is almost analogous.

Remark $2^{2}$. An example of an abelian group which satisfies the conditions (i) and (ii), but is not a free abelian group is given by Prof. L. Pontrjagin: Theory of topological commutative groups, Appendix I. Annals of Math. vol. 35 (1934).

To conclude this note the author wishes to thank Prof. Y. Akizuki, Prof. H. Anzai, Prof. J. Igusa and Prof. M. Takahashi for their valuable suggestions and advices.

2) Another kind of counter-examples were given by Prof. Akizuki. 